IMA Journal of Applied Mathematics (2018) Page 1 of 17

doi:10.1093/imamat/xxx000

\title{
Slip flow through channels with varying elliptic cross section
}

\author{
Z. M. Wilmott, C. J. W. Breward, S. J. Chapman
}

[Received on 14 May 2018]

\begin{abstract}
The low Reynolds number fluid flow through a channel with varying elliptic cross section is studied, with the inclusion of slip on the fluid-solid interface. An analytical expression for the flux through the channel is obtained, assuming that the slip length is small in comparison to the width of the channel, and a simplified expression for the flux in the limit of small eccentricity is also derived. Numerical results show that there is excellent agreement between the analytical expressions and the finite element solution for three examples presented in this paper. The expression for the flux is reformulated in terms of the semi-major and semi-minor radii, and the area and circumference, in order to estimate the flow through irregular channels. It is shown that the resulting expressions are extensions to existing estimation methods, the 'Aissen approximation' and 'hydraulic radius' methods, to include the effect of slip flow through channels with non-uniform cross section. Numerical results show that there is good agreement between the suggested flux estimation methods and the finite element solution for a pore throat extracted from a Berea sandstone. Furthermore, the suggested methods significantly outperform two common methods used to estimate the flux through an irregular channel, the 'volume matching' and 'minimum radius' methods.
\end{abstract}

Keywords: slip flow, low Reynolds flow, elliptic channel

\section{Introduction}

Many systems of practical interest, such as the low Reynolds number flow of oil through a pore throat in an oil reservoir (Wilmott et al., 2018), exhibit slip flow through an irregular channel. In order to understand how these systems behave, it is important to be able to calculate the flux through the channel in an efficient manner. However, due to the complexity of the problem, for channels with irregular geometry the only way to accurately compute the flux is to numerically solve the Stokes equations inside the channel, typically using a finite element or lattice Boltzmann method. This can be time consuming and computationally expensive, particularly if an application requires the flux through numerous channels, such as in a network model of a porous rock.

A common approach used to estimate the flux, without having to resort to expensive computations, is to suppose that there is an equivalent circularly cylindrical channel, with a radius to be determined, such that the flux through the cylindrical channel behaves similarly to the flux through the irregular channel. However, estimates for the effective radius are typically quite crude, such as taking the radius of the largest sphere which passes through the channel (Thovert et al., 1993), or by choosing the radius to be such that volume of the cylindrical channel is the same as the volume of the irregular channel (Al-Kharusi \& Blunt, 2008). Crude methods such as these are prone to systematic errors. For example, taking the radius to be given by the radius of the largest sphere which passes through the channel will consistently underestimate the flux. For large systems of channels, as might be used to model a porous medium, this leads to significant errors in estimating the permeability.

A more sophisticated approach is to estimate the flow by considering a channel with varying circular cross section, with the radius at each point along the length of the channel given by the largest inscribed sphere at that point along the length of the irregular channel (Ghosal, 2002). Since the radius at each point needs to be calculated anyway in order to find the radius at the most constricted point, this method requires negligible extra computational effort to extract the relevant data. However, more work is required to determine the flux through a channel with varying circular cross section than a channel with uniform cross section.

One disadvantage of assuming that the irregular geometry can be approximated by a channel with varying

(C) The author 2018. Published by Oxford University Press on behalf of the Institute of Mathematics and its Applications. All rights reserved. 
circular cross section is that, at each point along the length of the channel, there is only a single degree of freedom, the radius of the circle. This means that the circumference and area of the cross section cannot be varied independently, which makes estimating the flux particularly difficult for problems involving partial slip along the fluid-solid interface, since the flux depends primarily on the cross-sectional area, whereas the effect of slip is intimately related to the circumference of the cross section of the channel.

This motivates the study of flow through elliptical channels with varying cross section. By considering an elliptical cross section, as opposed to a circular cross section, we introduce two degrees of freedom and are therefore able to vary the area and circumference of the channel independently. We consider a model in which the slip length is small in comparison with the size of the channel, which is often applicable to systems in which the slip is caused by thin surface films.

Similar problems have been considered in the literature. Bahrami et al. (2009) study slip flow through channels with uniform circular, rectangular, trapezoidal, and double-trapezoidal cross sections. They consider an averaged slip boundary condition, so that the velocity of the fluid at the boundary is proportional to the average of the shear stress over the perimeter of the channel. The difference between the bulk velocity and the boundary velocity satisfies Stokes' equations with homogeneous boundary conditions. They determine a relationship between the Franning friction coefficient in the slip regime and the no-slip regime, which can be used to determine the flux. Ghosal (2002) studies flow through channels with slowly varying cross section, including slip due to electro-osmotic effects, assuming that the slip length is small in comparison to the dimensions of the channel. He solves the problem for an arbitrary cross section in terms of the Greens function, and finds expressions for the particular cases of slowly varying circular cross section and slowly varying rectangular cross section. In his model, the slip boundary condition is proportional to the electric field, and is independent of the shear stress at the boundary. Duan \& Muzychka (2007) study slip flow through an elliptical pipe with uniform cross section. They assume that the slip length is $O(1)$, and they use a binomial series expansion to approximate the metric in order to evaluate the flux.

In this paper, we study the problem of slip flow through a channel of varying elliptic cross section, with a straight central axis. Our aim is to determine the flux through the channel as a function of the slip length, assuming that the slip length is small in comparison to the dimensions of the channel. Furthermore, we assume that the channel is slender, and that the cross section varies slowly by comparison to its diameter. Our aim is to estimate the flux through irregularly shaped channels using an elliptic approximation, in order to be able to more efficiently compute the flow rate through pore throats in porous media.

In Section 2, we present a model for the velocity of the fluid in the channel. We assume that the velocity on the surface of the channel is proportional to the shear stress, with the constant of proportionality given by the slip length. In Section 3.1 we determine the flux through the channel to first order in the slip length. In Section 3.2 we determine the flux in the limit in which the eccentricity of the elliptic cross section is small. In Section 3.3 we compute the flux through three example channels and compare the equations for the flux given in Section 3.1 and 3.2 with the finite element solution. We compare the results with the estimations given by the minimum radius and volume matching methods.

In Section 4 we discuss popular methods for estimating the flux through irregular channels. We compare these methods with the elliptic approximation approach by computing the flux through a pore throat extracted from a sample of Berea sandstone using X-ray microtomography. Finally, in Section 5 we discuss our findings and their applicability to real-world problems.

\section{Model formulation}

We consider flow through a channel of varying elliptic cross section with a straight central axis, denoting the interior domain of the channel by $\Omega$, and the boundary by $\partial \Omega$. We suppose that the channel has length $L$, and that $R$ is the largest value of the major radius. The fluid is subject to a pressure difference, $P$, across the ends of the channel, and the surface allows partial slip, with slip length $\lambda$, such that $\lambda \ll R \ll L$.

We suppose that the origin of our coordinate system is at the mid point of one end of the channel, with the central axis of the channel lying along the $z$ axis, and we further suppose that the semi-major axis of the elliptic cross section in the $(x, y)$ plane for a given $z$ is at an angle $\phi(z)$ to the $x$ axis, as shown in Figure 1. 


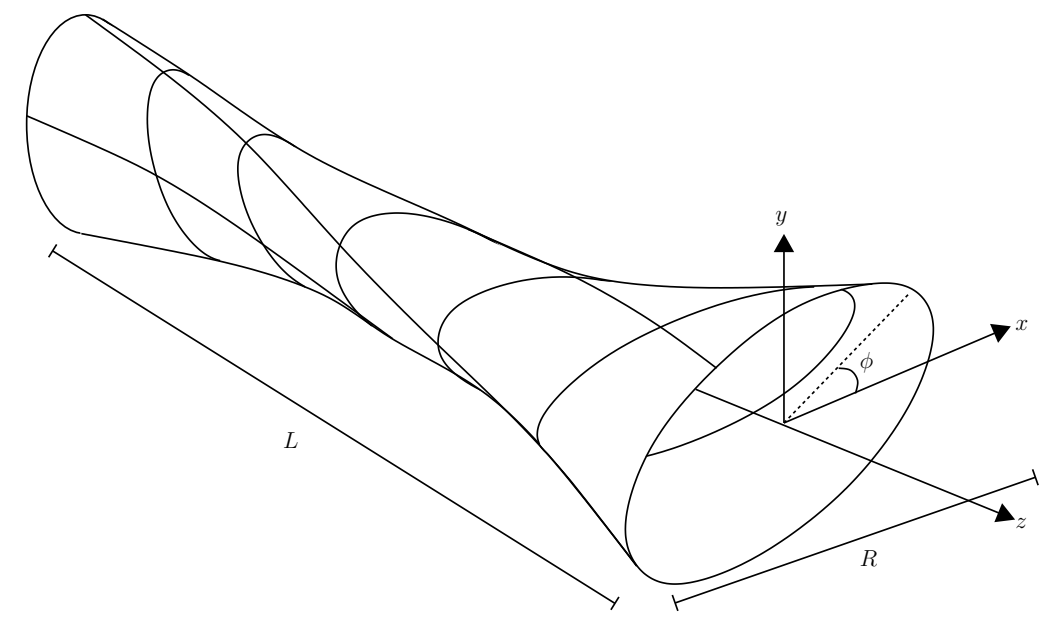

FIG. 1: Schematic diagram of the channel with varying elliptic cross section.

We suppose that $\phi$ varies slowly along the length of the channel, that is, $\phi_{z}=O(1 / L)$. For rapidly varying $\phi$, the torsion of the channel needs to be considered, however this is beyond the scope of this paper.

\subsection{Governing equations}

We model the flow in the channel using the incompressible Stokes equations,

$$
\mu \nabla^{2} \mathbf{u}=\nabla p, \quad \nabla \cdot \mathbf{u}=0
$$

where $\mathbf{u}$ is the velocity of the fluid, $p$ is the pressure, and $\mu$ is the viscosity of the fluid.

We prescribe a pressure difference, $P$, across the ends of the channel, and we suppose that the fluid satisfies a partial slip boundary condition on the fluid-solid interface, so that the tangential velocity of the fluid on the surface is proportional to the shear stress. Thus, $\mathbf{u}$ and $p$ satisfy the boundary conditions

$$
\begin{aligned}
& p=P, \quad \text { at } z=0, \\
& p=0, \quad \text { at } z=L, \\
& \mathbf{u} \cdot \mathbf{t}_{1,2}=\lambda \mathbf{n} \cdot\left(\nabla \mathbf{u}+(\nabla \mathbf{u})^{T}\right) \cdot \mathbf{t}_{1,2}, \quad \mathbf{u} \cdot \mathbf{n}=0, \quad \text { on } \partial \Omega,
\end{aligned}
$$

where $\mathbf{t}_{1}, \mathbf{t}_{2}$ and $\mathbf{n}$ are two tangential vectors and the normal vector, respectively, on the surface of the tube, $\partial \Omega$. We define the surface of the channel by $f(x, y, z)=0$, so that the tangential and normal vectors on the surface are given by

$$
\begin{aligned}
\mathbf{t}_{1} & =\frac{1}{\sqrt{\left(f_{x}^{2}+f_{y}^{2}\right)\left(f_{x}^{2}+f_{y}^{2}+f_{z}^{2}\right)}}\left[-f_{x} f_{z} \mathbf{i}-f_{y} f_{z} \mathbf{j}+\mathbf{k}\left(f_{x}^{2}+f_{y}^{2}\right)\right], \\
\mathbf{t}_{2} & =\frac{1}{\sqrt{f_{x}^{2}+f_{y}^{2}}}\left[f_{y} \mathbf{i}-f_{x} \mathbf{j}\right] \\
\mathbf{n} & =\frac{1}{\sqrt{f_{x}^{2}+f_{y}^{2}+f_{z}^{2}}}\left[f_{x} \mathbf{i}+f_{y} \mathbf{j}+f_{z} \mathbf{k}\right],
\end{aligned}
$$

where $\mathbf{i}, \mathbf{j}, \mathbf{k}$ are the basis vectors of the cartesian coordinate system. 
4 of 17

\subsection{Nondimensionalisation}

We nondimensionalise with the following scalings,

$$
\begin{gathered}
x=R \hat{x}, \quad y=R \hat{y}, \quad z=L \hat{z}, \\
p=P \hat{p}, \quad u^{*}=\varepsilon_{1} U \hat{u}^{*}, \quad v^{*}=\varepsilon_{1} U \hat{v}^{*}, \quad w^{*}=U \hat{w}^{*},
\end{gathered}
$$

where $U=P R^{2} / \mu L, \varepsilon_{1}=R / L, \mathbf{u}=u^{*} \mathbf{i}+v^{*} \mathbf{j}+w^{*} \mathbf{k}$, the star superscript denotes cartesian coordinates, and the hat denotes the dimensionless variable.

We define the dimensionless slip length to be $\varepsilon_{2}=\lambda / R \ll 1$. For a typical pore throat, $L \sim 10^{-5} \mathrm{~m}$, $R \sim 10^{-6} \mathrm{~m}$, and $\lambda \sim 10^{-9} \mathrm{~m}$, and thus $\varepsilon_{1}=10^{-1}$ and $\varepsilon_{2}=10^{-3}$. We find that, neglecting quadratic terms in $\varepsilon_{1}$ and $\varepsilon_{2}$, equations (2.1) - (2.4) read

$$
\begin{gathered}
w_{x x}^{*}+w_{y y}^{*}=p_{z}, \quad p_{x}=0, \quad p_{y}=0, \\
u_{x}^{*}+v_{y}^{*}+w_{z}^{*}=0,
\end{gathered}
$$

subject to

$$
\begin{array}{rlrl}
p & =1, & \text { at } z=0, \\
p & =0, & \text { at } z=1, \\
w^{*} & =\frac{\varepsilon_{2}}{\sqrt{f_{x}^{2}+f_{y}^{2}}}\left(w_{x}^{*} f_{x}+w_{y}^{*} f_{y}\right), & & \text { at } f=0, \\
u^{*} f_{y}-v^{*} f_{x} & =\frac{\varepsilon_{2}}{\sqrt{f_{x}^{2}+f_{y}^{2}}}\left[\left(f_{y}^{2}-f_{x}^{2}\right)\left(u_{y}^{*}+v_{x}^{*}\right)\right. & & \\
& \left.+2 f_{x} f_{y}\left(u_{x}^{*}-v_{y}^{*}\right)+f_{z}\left(w_{x}^{*} f_{y}-w_{y}^{*} f_{x}\right)\right], & \text { at } f=0, \\
f_{x} u^{*}+f_{y} v^{*}+f_{z} w^{*}= & 0, & & \text { at } f=0,
\end{array}
$$

where we have dropped the hat notation for convenience.

\subsection{Elliptic coordinates}

We assume that, for each value of $z$, the cross section of the channel in the $(x, y)$ plane is an ellipse, with semi-major and semi-minor radii given by $r_{a}(z)$ and $r_{b}(z)$ respectively. Hence, the surface of the channel is given by

$$
f(x, y, z)=1-\left(\frac{x \cos \phi+y \sin \phi}{r_{a}}\right)^{2}-\left(\frac{y \cos \phi-x \sin \phi}{r_{b}}\right)^{2}=0 .
$$

In order to simplify the boundary conditions $(2.14)-(2.16)$, we formulate the problem in terms of elliptic coordinates $(\eta, \xi, z)$ using the change of variables

$$
\begin{aligned}
& x=a(z) \cosh \eta \cos \xi \cos \phi-a(z) \sinh \eta \sin \xi \sin \phi, \\
& y=a(z) \cosh \eta \cos \xi \sin \phi+a(z) \sinh \eta \sin \xi \cos \phi,
\end{aligned}
$$

where $\eta \in[0, \infty)$ and $\xi \in[0,2 \pi)$. The foci of the ellipse in the $(x, y)$ plane passing through $z$ are given by $x= \pm a(z) \cos \phi, y= \pm a(z) \sin \phi$. In the $(\eta, \xi, z)$ coordinate system, the boundary of the tube is given by $\eta=N(z)$, where $a(z)$ and $N(z)$ are given by $a=\sqrt{r_{a}^{2}-r_{b}^{2}}$ and $N=\tanh ^{-1}\left(r_{b} / r_{a}\right)$.

We define $\mathbf{u}=u^{*} \mathbf{i}+v^{*} \mathbf{j}+w^{*} \mathbf{k}=u \mathbf{e}_{\eta}+v \mathbf{e}_{\xi}+w \mathbf{e}_{z}$, where $\left(\mathbf{e}_{\eta}, \mathbf{e}_{\xi}, \mathbf{e}_{z}\right)$ are the basis vectors in the elliptic coordinate system. In the $(\eta, \xi, z)$ coordinate system, equations $(2.10)-(2.16)$, read

$$
\begin{gathered}
\frac{\partial^{2} w}{\partial \eta^{2}}+\frac{\partial^{2} w}{\partial \xi^{2}}=a^{2} h^{2} \frac{\partial p}{\partial z}, \quad \frac{\partial p}{\partial \eta}=0, \quad \frac{\partial p}{\partial \xi}=0 \\
\frac{1}{a^{2} h^{2}}\left[\frac{\partial}{\partial \eta}(a h u)+\frac{\partial}{\partial \xi}(a h v)\right]+\frac{1}{a^{2}} \frac{\partial}{\partial z}\left(a^{2} w\right)=0
\end{gathered}
$$


subject to

$$
\begin{aligned}
& p=1, \quad \text { at } z=0, \\
& p=0, \quad \text { at } z=1 \text {, } \\
& w=\frac{-\varepsilon_{2}}{a h} \frac{\partial w}{\partial \eta}, \quad \text { at } \eta=N, \\
& a h \frac{\mathrm{d} N}{\mathrm{~d} z} w-u=0, \quad \text { at } \eta=N,
\end{aligned}
$$

where

$$
h=\sqrt{\frac{1}{2}(\cosh 2 \eta-\cos 2 \xi)} .
$$

We have omitted the elliptic form of (2.15) since we only need to determine $w$ to find the flux through the channel, and this boundary condition is only required to determine $u$ and $v$.

Since the problem is symmetric under a rotation of $\pi$, we require that the solution satisfies

$$
\frac{\partial w}{\partial \eta}=0, \quad \text { at } \eta=0
$$

\section{Solution}

\subsection{Flux through the channel}

Integrating (2.21) over the cross section of the channel, $A(z)$, yields

$$
\int_{0}^{2 \pi} \int_{0}^{N(z)}\left[\frac{\partial}{\partial \eta}(a h u)+\frac{\partial}{\partial \xi}(a h v)+\frac{\partial}{\partial z}\left(a^{2} h^{2} w\right)\right] \mathrm{d} \eta \mathrm{d} \xi=0 .
$$

By Leibniz's integral rule,

$$
\begin{aligned}
\int_{0}^{2 \pi} \int_{0}^{N(z)} \frac{\partial}{\partial z}\left(a^{2} h^{2} w\right) \mathrm{d} \eta \mathrm{d} \xi & =\frac{\mathrm{d}}{\mathrm{d} z} \int_{0}^{2 \pi} \int_{0}^{N(z)} a^{2} h^{2} w \mathrm{~d} \eta \mathrm{d} \xi-\int_{0}^{2 \pi} \frac{\mathrm{d} N}{\mathrm{~d} z}\left[a^{2} h^{2} w\right]_{\eta=N} \mathrm{~d} \xi \\
& =\frac{\mathrm{d}}{\mathrm{d} z} \iint_{A} w \mathrm{~d} A-\int_{0}^{2 \pi} \frac{\mathrm{d} N}{\mathrm{~d} z}\left[a^{2} h^{2} w\right]_{\eta=N} \mathrm{~d} \xi
\end{aligned}
$$

where we have used that $\mathrm{d} A:=\mathrm{d} x \mathrm{~d} y=a^{2} h^{2} \mathrm{~d} \eta \mathrm{d} \xi$.

Substituting (3.3) into (3.1) and applying Green's Theorem yields

$$
\frac{\mathrm{d}}{\mathrm{d} z} \iint_{A} w \mathrm{~d} A-\int_{0}^{2 \pi} \frac{\mathrm{d} N}{\mathrm{~d} z}\left[a^{2} h^{2} w\right]_{\eta=N} \mathrm{~d} \xi+\int_{\partial A} a h\left[u \frac{\mathrm{d} \xi}{\mathrm{d} s}-v \frac{\mathrm{d} \eta}{\mathrm{d} s}\right] \mathrm{d} s=0,
$$

Taking $s$ to be arc length along the circumference of the ellipse, $\mathrm{d} s=\sqrt{\mathrm{d} x^{2}+\mathrm{d} y^{2}}$, we find that $\frac{\mathrm{d} \eta}{\mathrm{d} s}=0$, and thus (3.4) reads

$$
\frac{\mathrm{d}}{\mathrm{d} z} \iint_{A} w \mathrm{~d} A+\int_{0}^{2 \pi}\left[a h u-a^{2} h^{2} w \frac{\mathrm{d} N}{\mathrm{~d} z}\right]_{\eta=N} \mathrm{~d} \xi=0 .
$$

By imposing (2.25), we obtain the conservation of flux equation

$$
\frac{\mathrm{d} Q}{\mathrm{~d} z}=0, \quad \text { where } \quad Q=\iint_{A} w \mathrm{~d} A .
$$

To compute the flux, we find $w$ by solving (2.20a) and (2.21) subject to (2.24) - (2.27). From (2.20) we see that $p=p(z)$. We expand $w$ and $p$ in powers of $\varepsilon_{2}$, to find that, at leading order, equations (2.20a) and (2.24) read

$$
\frac{\partial^{2} w^{(0)}}{\partial \eta^{2}}+\frac{\partial^{2} w^{(0)}}{\partial \xi^{2}}=\frac{a^{2}}{2} \frac{\mathrm{d} p^{(0)}}{\mathrm{d} z}(\cosh 2 \eta-\cos 2 \xi)
$$




\section{6 of 17}

subject to $w^{(0)}=0$ on $\eta=N$. A particular solution to the inhomogeneous equation (3.7) is

$$
w_{P}^{(0)}=\frac{a^{2}}{8} \frac{\mathrm{d} p^{(0)}}{\mathrm{d} z}(\cosh 2 \eta+\cos 2 \xi) .
$$

However, this does not satisfy the boundary condition $w^{(0)}=0$ on $\eta=N$. In order to rectify this issue, we use separation of variables to solve the complimentary homogeneous equation

$$
\frac{\partial^{2} w_{H}^{(0)}}{\partial \eta^{2}}+\frac{\partial^{2} w_{H}^{(0)}}{\partial \xi^{2}}=0
$$

observing that, by linearity, $w^{(0)}=w_{P}^{(0)}+w_{H}^{(0)}$ satisfies (3.7). By imposing (2.27), we find that $w_{H}^{(0)}$ is of the form

$$
w_{H}^{(0)}=\frac{1}{2} \alpha_{0}^{(0)}+\sum_{k=1}^{\infty}\left(\alpha_{k}^{(0)} \cos k \xi+\beta_{k}^{(0)} \sin k \xi\right) \cosh k \eta .
$$

Hence, by imposing $w^{(0)}=w_{P}^{(0)}+w_{H}^{(0)}=0$ on $\eta=N$, we find that

$$
w^{(0)}=\frac{a^{2}}{8} \frac{\mathrm{d} p^{(0)}}{\mathrm{d} z}\left[\cosh 2 \eta-\cosh 2 N+\cos 2 \xi-\frac{\cos 2 \xi \cosh 2 \eta}{\cosh 2 N}\right] .
$$

By writing this expression in cartesian coordinates, we find that

$$
w^{(0)}=-\frac{r_{a}^{2} r_{b}^{2}}{2\left(r_{a}^{2}+r_{b}^{2}\right)} \frac{\mathrm{d} p^{(0)}}{\mathrm{d} z}\left[1-\frac{(x \cos \phi+y \sin \phi)^{2}}{r_{a}^{2}}-\frac{(y \cos \phi-x \sin \phi)^{2}}{r_{b}^{2}}\right],
$$

which is the expression for quasi-Poiseuille flow through a channel with elliptic cross section. The leadingorder flux is given by

$$
Q^{(0)}=-\frac{r_{a}^{2} r_{b}^{2}}{2\left(r_{a}^{2}+r_{b}^{2}\right)} \frac{\mathrm{d} p^{(0)}}{\mathrm{d} z} \iint_{A}\left(1-\frac{(x \cos \phi+y \sin \phi)^{2}}{r_{a}^{2}}-\frac{(y \cos \phi-x \sin \phi)^{2}}{r_{b}^{2}}\right) \mathrm{d} x \mathrm{~d} y .
$$

We make the change of variables $x \cos \phi+y \sin \phi=r_{a} r \cos \theta, y \cos \phi-x \sin \phi=r_{b} r \sin \theta$, which yields

$$
Q^{(0)}=-\frac{r_{a}^{3} r_{b}^{3}}{2\left(r_{a}^{2}+r_{b}^{2}\right)} \frac{\mathrm{d} p^{(0)}}{\mathrm{d} z} \int_{0}^{2 \pi} \int_{0}^{1}\left(1-r^{2}\right) r \mathrm{~d} r \mathrm{~d} \theta=-\frac{\pi r_{a}^{3} r_{b}^{3}}{4\left(r_{a}^{2}+r_{b}^{2}\right)} \frac{\mathrm{d} p}{\mathrm{~d} z} .
$$

Solving (3.6) subject to (2.22) and (2.23), we find that

$$
\frac{\mathrm{d} p^{(0)}}{\mathrm{d} z}=-\frac{r_{a}^{2}+r_{b}^{2}}{r_{a}^{3} r_{b}^{3}}\left[\int_{0}^{1} \frac{r_{a}^{2}+r_{b}^{2}}{r_{a}^{3} r_{b}^{3}} \mathrm{~d} z\right]^{-1},
$$

and thus

$$
Q^{(0)}=\left\{\int_{0}^{1}\left[\frac{\pi r_{a}^{3} r_{b}^{3}}{4\left(r_{a}^{2}+r_{b}^{2}\right)}\right]^{-1} \mathrm{~d} z\right\}^{-1}
$$

We observe that the term inside the square brackets is the well-known expression for the flux through an elliptic pipe. Hence, the conductivity of the pipe with varying elliptic cross section is given by the harmonic mean of the conductivites of each cross section along the length of the pipe.

To find the contribution to the flux resulting from the partial slip along the surface, $Q^{(1)}$, we require the solution for the fluid velocity at the next order, $w^{(1)}$. From (2.20), we obtain the governing equation for $w^{(1)}$, which reads

$$
\frac{\partial^{2} w^{(1)}}{\partial \eta^{2}}+\frac{\partial^{2} w^{(1)}}{\partial \xi^{2}}=\frac{a^{2}}{2} \frac{\mathrm{d} p^{(1)}}{\mathrm{d} z}(\cosh 2 \eta-\cos 2 \xi)
$$


We separate the fluid velocity into a particular solution and a solution to the homogeneous problem, given by

$$
\begin{aligned}
& w_{P}^{(1)}=\frac{a^{2}}{8} \frac{\mathrm{d} p^{(1)}}{\mathrm{d} z}(\cosh 2 \eta+\cos 2 \xi), \\
& w_{H}^{(1)}=\frac{1}{2} \alpha_{0}^{(1)}+\sum_{k=1}^{\infty}\left(\alpha_{k}^{(1)} \cos k \xi+\beta_{k}^{(1)} \sin k \xi\right) \cosh k \eta .
\end{aligned}
$$

The first-order terms in (2.24) read

$$
\begin{aligned}
\frac{a^{2}}{8} \frac{\mathrm{d} p^{(1)}}{\mathrm{d} z}(\cosh 2 N+\cos 2 \xi)+\frac{1}{2} \alpha_{0}^{(1)}+\sum_{k=1}^{\infty}\left(\alpha_{k}^{(1)} \cos k \xi\right. & \left.+\beta_{k}^{(1)} \sin k \xi\right) \cosh k N \\
& =-\frac{a \sinh 2 N}{2 \sqrt{2} \cosh 2 N} \frac{\mathrm{d} p^{(0)}}{\mathrm{d} z} \sqrt{\cosh 2 N-\cos 2 \xi}
\end{aligned}
$$

where the Fourier coefficients are given by $\beta_{k}^{(1)}=0$, and

$$
\begin{aligned}
& \alpha_{0}^{(1)}=-\frac{a^{2}}{4} \cosh 2 N \frac{\mathrm{d} p^{(1)}}{\mathrm{d} z}-\frac{a \sinh 2 N}{2 \sqrt{2} \pi \cosh 2 N} \frac{\mathrm{d} p^{(0)}}{\mathrm{d} z} \int_{0}^{2 \pi} \sqrt{\cosh 2 N-\cos 2 \xi} \mathrm{d} \xi \\
& \alpha_{2}^{(1)}=-\frac{a^{2}}{8 \cosh 2 N} \frac{\mathrm{d} p^{(1)}}{\mathrm{d} z}-\frac{a \sinh 2 N}{2 \sqrt{2} \pi \cosh ^{2} 2 N} \frac{\mathrm{d} p^{(0)}}{\mathrm{d} z} \int_{0}^{2 \pi} \cos 2 \xi \sqrt{\cosh 2 N-\cos 2 \xi} \mathrm{d} \xi \\
& \alpha_{k}^{(1)}=-\frac{a \sinh 2 N}{2 \sqrt{2} \pi \cosh 2 N \cosh k N} \frac{\mathrm{d} p^{(0)}}{\mathrm{d} z} \int_{0}^{2 \pi} \cos k \xi \sqrt{\cosh 2 N-\cos 2 \xi} \mathrm{d} \xi
\end{aligned}
$$

for $k \neq 0,2$. We are unable to evaluate the integrals in (3.21) - (3.23), and thus $w^{(1)}$ is given as an infinite series of integrals. However, we can make further progress with the first-order flux, and we find that

$$
\begin{aligned}
Q^{(1)} & =\frac{a^{2}}{2} \int_{0}^{N} \int_{0}^{2 \pi}\left(w_{P}^{(1)}+w_{H}^{(1)}\right)(\cosh 2 \eta-\cos 2 \xi) \mathrm{d} \xi \mathrm{d} \eta \\
& =\frac{a^{4} \pi}{32} \sinh 2 N \cosh 2 N \frac{\mathrm{d} p^{(1)}}{\mathrm{d} z}+\frac{a^{2} \pi}{4} \sinh 2 N\left(\alpha_{0}^{(1)}-\alpha_{2}^{(1)}\right) .
\end{aligned}
$$

Substituting (3.21) and (3.22) yields

$$
Q^{(1)}=-\frac{a^{4} \pi}{32} \tanh 2 N \sinh ^{2} 2 N \frac{\mathrm{d} p^{(1)}}{\mathrm{d} z}-\frac{a^{3}}{8 \sqrt{2}} \tanh ^{2} 2 N \frac{\mathrm{d} p^{(0)}}{\mathrm{d} z} \int_{0}^{2 \pi}(\cosh 2 N-\cos 2 \xi)^{3 / 2} \mathrm{~d} \xi .
$$

We write (3.26) in terms of $r_{a}$ and $r_{b}$, and substitute (3.15) to obtain

$$
Q^{(1)}=-\frac{\pi r_{a}^{3} r_{b}^{3}}{4\left(r_{a}^{2}+r_{b}^{2}\right)} \frac{\mathrm{d} p^{(1)}}{\mathrm{d} z}+\frac{4 \int_{0}^{2 \pi}\left(r_{a}^{2} \sin ^{2} \theta+r_{b}^{2} \cos ^{2} \theta\right)^{3 / 2} \mathrm{~d} \theta}{\pi r_{a} r_{b}\left(r_{a}^{2}+r_{b}^{2}\right)}\left[\int_{0}^{1}\left(\frac{\pi r_{a}^{3} r_{b}^{3}}{4\left(r_{a}^{2}+r_{b}^{2}\right)}\right)^{-1}\right]^{-1}
$$

From (3.6) we find that $Q^{(1)}$ is constant, so, by integrating (3.27) from $z=0$ to $z=1$ and imposing (2.22) and (2.23), we find that the first-order component of the flux is given by

$$
Q^{(1)}=\frac{\int_{0}^{1}\left[\left(\frac{\pi^{2}}{16} r_{a}^{4} r_{b}^{4}\right)^{-1} \int_{0}^{2 \pi}\left(r_{a}^{2} \sin ^{2} \theta+r_{b}^{2} \cos ^{2} \theta\right)^{3 / 2} \mathrm{~d} \theta\right] \mathrm{d} z}{\left(\int_{0}^{1}\left[\frac{\pi r_{a}^{3} r_{b}^{3}}{4\left(r_{a}^{2}+r_{b}^{2}\right)}\right]^{-1} \mathrm{~d} z\right)^{2}} .
$$




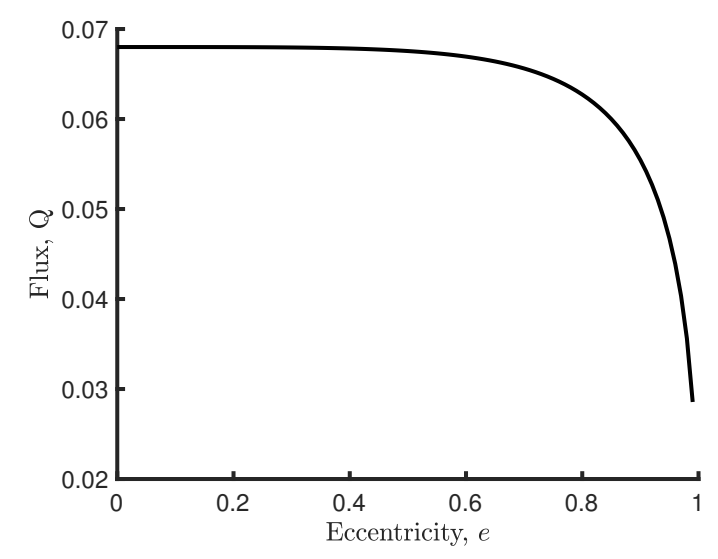

FIG. 2: Plot of the flux, given by (3.29), through a uniform channel of elliptic cross section against the eccentricity.

Combining equations (3.16) and (3.28) yields the total flux to order $\varepsilon_{2}$, which reads

$$
Q=\left\{\int_{0}^{1}\left[\frac{\pi r_{a}^{3} r_{b}^{3}}{4\left(r_{a}^{2}+r_{b}^{2}\right)}\left(1+\frac{4 \varepsilon_{2}}{\pi r_{a} r_{b}\left(r_{a}^{2}+r_{b}^{2}\right)} \int_{0}^{2 \pi}\left(r_{a}^{2} \sin ^{2} \theta+r_{b}^{2} \cos ^{2} \theta\right)^{3 / 2} \mathrm{~d} \theta\right)\right]^{-1} \mathrm{~d} z\right\}^{-1}+O\left(\varepsilon_{2}^{2}\right) .
$$

As for the leading-order flux, the total flux is the harmonic mean of the equivalent flux through each cross section of the channel. Note that, since we assumed that the rotation of the elliptical cross section varies slowly along the length of the channel, $\phi$ does not appear in (3.29). In Figure 2, we plot the the flux, $Q$, given by (3.29), through a uniform elliptical channel with cross-sectional area $\pi r_{a} r_{b}=1$ against the eccentricity, $e=\sqrt{1-r_{b}^{2} / r_{a}^{2}}$, where we have taken $\varepsilon_{2}=0.1$. As the eccentricity increases, the total flux through the channel decreases due to stagnant flow near the outermost edges of the ellipse.

\subsection{Solution for small eccentricity}

It is possible to write the integral in $\theta$ in (3.29) in terms of elliptic integrals of the first and second kind. However, for certain applications it may be impractical to compute these for each cross section. In the case where $\delta=\left(r_{a}^{2}-r_{b}^{2}\right) /\left(r_{a}^{2}+r_{b}^{2}\right) \ll 1$, which corresponds the eccentricity of the ellipse being small, we are able to simplify the integral. To exploit this small parameter, we make the change of variable

$$
\cos ^{2} \theta=\frac{1+\zeta}{2}, \quad \sin ^{2} \theta=\frac{1-\zeta}{2},
$$

so that the integral reads

$$
\int_{0}^{2 \pi}\left(r_{a}^{2} \sin ^{2} \theta+r_{b}^{2} \cos ^{2} \theta\right)^{3 / 2} \mathrm{~d} \theta=\frac{\left(r_{a}^{2}+r_{b}^{2}\right)^{3 / 2}}{\sqrt{2}} \int_{-1}^{1} \frac{(1-\delta \zeta)^{3 / 2}}{\sqrt{1-\zeta^{2}}} \mathrm{~d} \zeta
$$

We expand the integrand in powers of $\delta$ and perform the integration to find that

$$
\int_{0}^{2 \pi}\left(r_{a}^{2} \sin ^{2} \theta+r_{b}^{2} \cos ^{2} \theta\right)^{3 / 2} \mathrm{~d} \theta \sim \frac{\pi\left(r_{a}^{2}+r_{b}^{2}\right)^{3 / 2}}{\sqrt{2}}+O\left(\delta^{2}\right)
$$




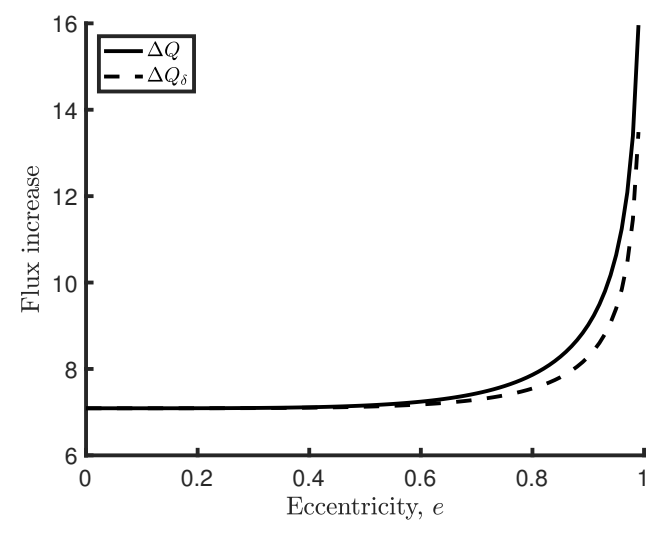

(a) Relative flux increase due to slip

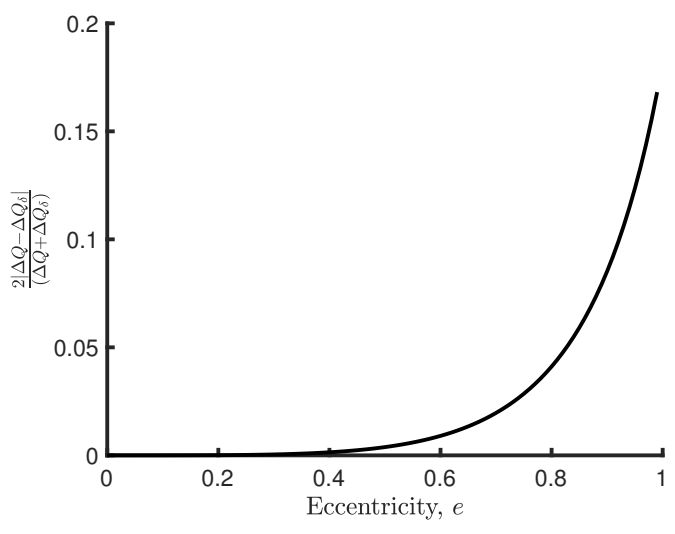

(b) Difference between $\Delta Q$ and $\Delta Q_{\delta}$

FIG. 3: Comparison between the flux increase due to slip for $Q$, given by (3.29), and $Q_{\delta}$, given by (3.33), showing a) the relative flux increase for the two solutions as a function of the eccentricity, and b) the difference in the relative flux increase between the two solutions as a function of the eccentricity, where $\Delta Q=Q_{1} / Q_{0}$, and $\Delta Q_{\delta}=Q_{1 \delta} / Q_{0 \delta}$.

By substituting (3.32) into (3.29), we find the flux through the channel can be approximated by

$$
Q_{\delta} \sim Q_{\delta}^{(0)}+\varepsilon_{2} Q_{\delta}^{(1)} \sim\left\{\int_{0}^{1}\left[\frac{\pi r_{a}^{3} r_{b}^{3}}{4\left(r_{a}^{2}+r_{b}^{2}\right)}\left(1+\frac{2 \sqrt{2} \varepsilon_{2} \sqrt{r_{a}^{2}+r_{b}^{2}}}{r_{a} r_{b}}\right)\right]^{-1} \mathrm{~d} z\right\}^{-1}
$$

In Figure 3a, we compare the relative flux increase due to slip given by the full expression, $\Delta Q:=Q^{(1)} / Q^{(0)}$, and the approximation, $\Delta Q_{\delta}:=Q^{(1)}{ }_{\delta} / Q^{(0)}$, against the eccentricity, $e$. We plot the solution for a uniform elliptic channel with cross-sectional area given by $\pi r_{a} r_{b}=1$. As the eccentricity increases, we see that the relative flux increase due to slip also increases. This is because the circumference to area ratio of the elliptic cross section increases, and channels with a greater surface area are more greatly affected by slip along the boundary.

In Figure 3b, we plot the difference between the relative flux increase given by the two expressions, $\Delta Q$ and $\Delta Q_{\delta}$, calculated using $2\left|\Delta Q-\Delta Q_{\delta}\right| /\left(\Delta Q+\Delta Q_{\delta}\right)$. We see that the difference is greatest (16.8\%) for $e=1$, and decreases to zero as $e \rightarrow 0$, with the error less than $1 \%$ for $e<0.62$.

\subsection{Comparison between flux estimation methods}

A common method used to estimate the flux through a non-uniform channel is to assume that the channel can be approximated by a circular cylinder with a 'hydraulic radius' to be determined. Two popular estimates for the hydraulic radius are the radius of the inscribed circle at the most constricted point along the channel, which we will call the 'minimum radius' method, and the radius such that the volume of the cylinder is the same as the volume of the channel, which we will call the 'volume matching' method. We compare the flux computed using these approximations with the fluxes given by (3.29) and (3.33) for three channels with varying elliptic cross section, shown in Figure 4. The geometries of the three channels are chosen to study how accurately the different methods estimate the flux through a channel with i) high eccentricity, ii) a bottleneck region, and iii) high torsion. For each channel, the aspect ratio, $\varepsilon_{1}$, the minimum and maximum eccentricity along the length of the channel, $e_{\min }$ and $e_{\max }$, respectively, and the minimum and maximum value of $\delta=e^{2} /\left(2-e^{2}\right)$ along the length of the channel, $\delta_{\min }$ and $\delta_{\max }$, respectively, are given in Table 1 . 


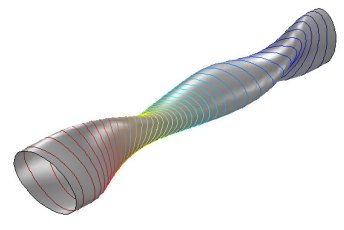

(a) Channel 1

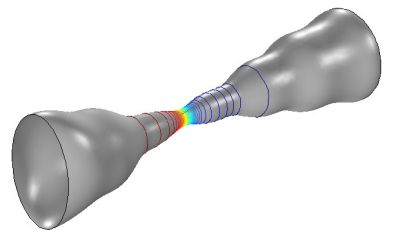

(b) Channel 2

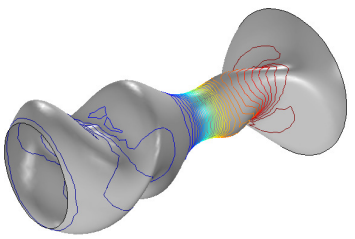

(c) Channel 3

FIG. 4: Diagrams of the three channels used to compare the flux estimation methods.

\begin{tabular}{c|c|c|c|c|c} 
& $\varepsilon_{1}$ & $e_{\min }$ & $e_{\max }$ & $\delta_{\min }$ & $\delta_{\min }$ \\
\hline \hline Channel 1 & 0.073 & 0.75 & 0.92 & 0.39 & 0.72 \\
Channel 2 & 0.14 & 0 & 0.71 & 0 & 0.34 \\
Channel 3 & 0.28 & 0 & 0.95 & 0 & 0.83
\end{tabular}

Table 1: Dimensionless parameter values for the three pore channels.

We also consider two 'intermediate' approaches in which the flux is estimated by approximating the geometry of the channel with a channel of varying circular cross section. The first involves computing the flux using (3.29), with $r_{a}=r_{b}=r$ being the radius of the maximum inscribed circle at each cross section, and the second involves computing the flux using (3.29), with $r_{a}=r_{b}=r$ being the radius of the minimum circumscribed circle at each cross section. We call these the 'inscribed circle' and 'circumscribed circle' methods, respectively. Both of these approximations improve on the minimum radius and volume matching methods because they take into consideration the variation of the channel cross section along its length, but they are easier to compute than the elliptic approximation because only one length needs to be found at each cross section. We compare the flux calculated using the different methods with the numerical finite element solution to $(2.1)-(2.4)$, which we take to be our 'true' solution.

In Figure 5a, we plot the total flux through channel 1 against the slip length. The 'minimum radius', 'volume matching', 'inscribed circle', and 'circumscribed circle' methods are labelled accordingly, and we also plot the solutions for $Q$ and $Q_{\delta}$, given by (3.29) and (3.33), respectively. Since the elliptical cross section is rotating slowly, the effects of torsion are minimal and thus we see that there is good agreement between $Q$ and the finite element solution. The elliptical solution with small eccentricity, $Q_{\delta}$ is nearly indistinguishable from $Q$, despite the eccentricity of the channel being relatively high, since the error for this solution is $O\left(\varepsilon_{2} \delta^{2}\right)$. The volume matching method is the next most accurate model, but overestimates the total flux. The circumscribed circle solution significantly overestimates the flux, and the inscribed circle and minimum radius method significantly underestimate the flux.

In Figure 5b, we plot the relative flux increase due to slip, $Q_{1} / Q_{0}$, against the slip length for channel 1. We observe that there is excellent agreement between $Q$ and the finite element solution, with $Q_{\delta}$ being the next most accurate. The volume matching method and inscribed circle method slightly underestimate and overestimate, respectively, the flux increase. As for the total flux, the minimum radius and circumscribed circle methods are the least accurate.

In Figure 6a we plot the total flux through channel 2 against the slip length. We see that $Q, Q_{\delta}$, and the inscribed and circumscribed circle solutions provide excellent estimations for the flux. However, all of these methods slightly overestimate the flux due to the torsion of the channel reducing the flow rate. The minimum radius and volume matching methods are the least accurate. In Figure $6 \mathrm{~b}$ we plot the relative flux increase due to slip against the slip length. Again, $Q, Q_{\delta}$, and the inscribed and circumscribed circle solutions are in 


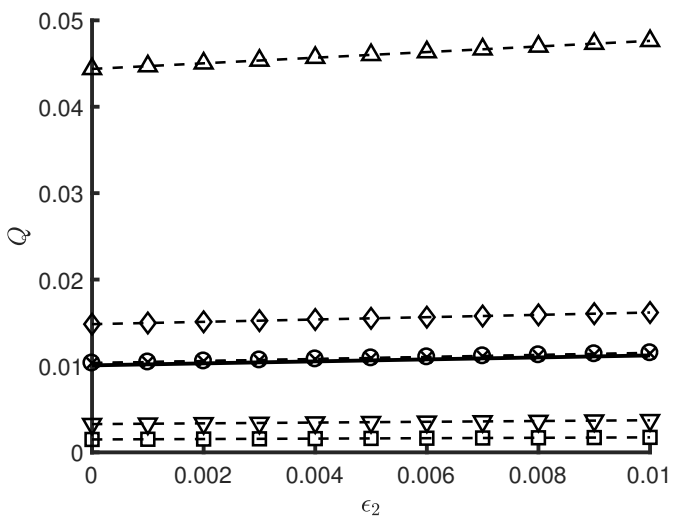

(a) Total flux

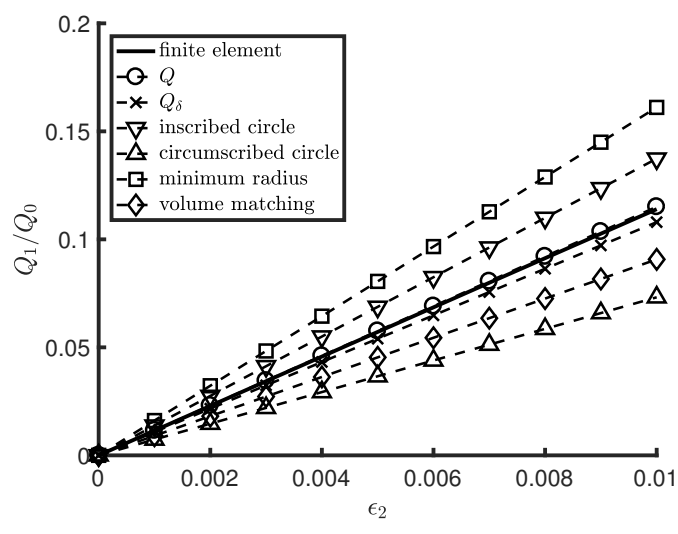

(b) Relative flux increase due to slip

FIG. 5: Comparison between the different flux estimation methods for channel 1, showing a) the total flux through channel 1 , and $b$ ) the relative flux increase in the presence of partial slip along the surface of the channel.

excellent agreement with the finite element solution, and the minimum radius and volume matching methods significantly overestimate and underestimate, respectively, the flux increase.

In Figure 7a, we plot the total flux against the slip length for channel 3, and in Figure $7 \mathrm{~b}$ we plot the relative increase in flux due to slip against the slip length. As for the other examples, the elliptic solutions, $Q$ and $Q_{\delta}$, provide the best estimate for the total flux, but overestimate the flux due to the torsion of the channel decreasing the flow rate. These methods also provide the best estimate for the flux increase. The two methods often used in practice, the volume matching and minimum radius method, significantly overestimate and underestimate the flux, respectively.

These examples demonstrate the care that needs to be taken when applying the minimum radius or volume matching methods. The minimum radius method appears to consistently underestimate the total flux and overestimate the relative flux increase as the slip increases, and the volume matching method appears to consistently overestimate the total flux and underestimate the relative flux increase. We see that, across the three channels, $Q$ and $Q_{\delta}$ typically give the best fit to the finite element solution. This is to be expected, since the channels considered have an elliptic cross section. However, there are many applications for which fluid moves through a channel which does not have an elliptical cross section. In Section 4, we discuss one such application, constructing a network model from a rock sample, and compare the different methods on flow through a pore throat extracted from a rock sample using X-ray microtomography.

\section{Flow through irregularly-shaped channels}

Modelling porous structures as networks is a popular way of studying flow through porous media (Al-Kharusi \& Blunt, 2008; Al-Raoush \& Willson, 2005; Sheng \& Thompson, 2016). This is due to the computational efficiency of this approach, and the relative ease with which multiple representative networks can be generated once the properties of a particular porous substance are known. This method is sometimes used within the oil industry to study flow through reservoir rock, since performing multiple experiments can be time-consuming and expensive. Additionally, by using a computational model, the effect of different conditions, such as oil composition, brine composition, and injection rate, can be tested on the same sample. In particular, it has been suggested that the salinity of the brine in a reservoir affects the thickness of the thin water film separating the oil phase from the solid phase (Lager et al., 2008; Lee et al., 2010; Myint \& Firoozabadi, 2015). This results in partial slip along the oil-solid interface (Wilmott et al., 2018). Thus, in order to create an accurate network representation of a rock sample to be used for low salinity waterflooding models, it is important to 


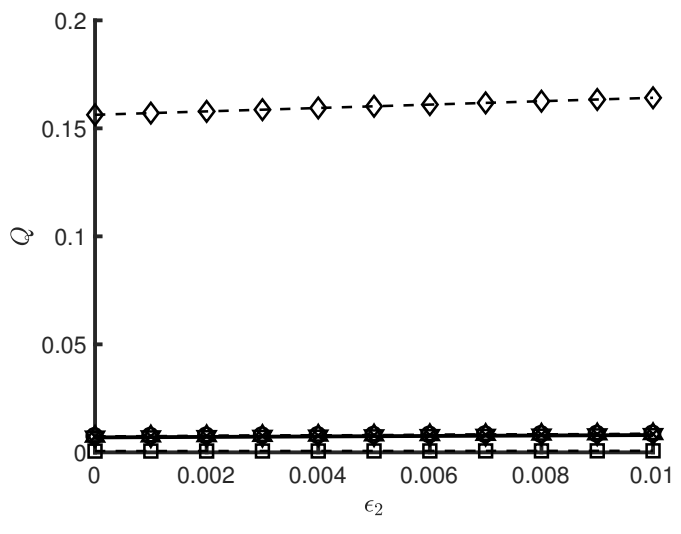

(a) Total flux

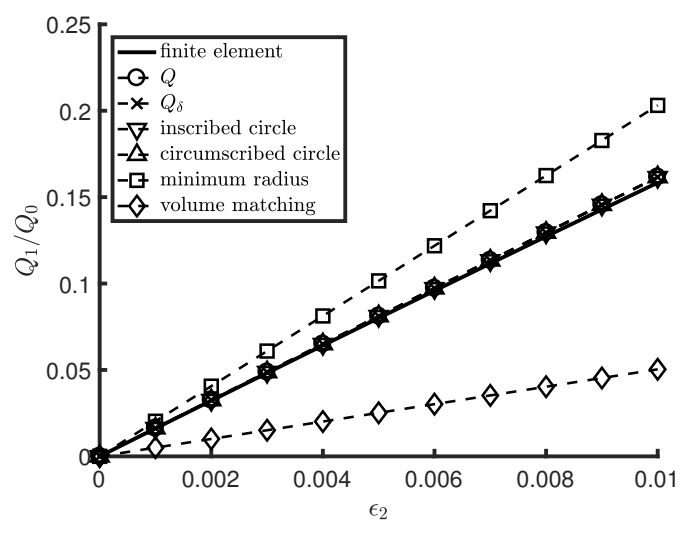

(b) Relative flux increase due to slip

FIG. 6: Comparison between the different flux estimation methods for channel 2, showing a) the total flux through channel 2, and b) the relative flux increase in the presence of partial slip along the surface of the channel.

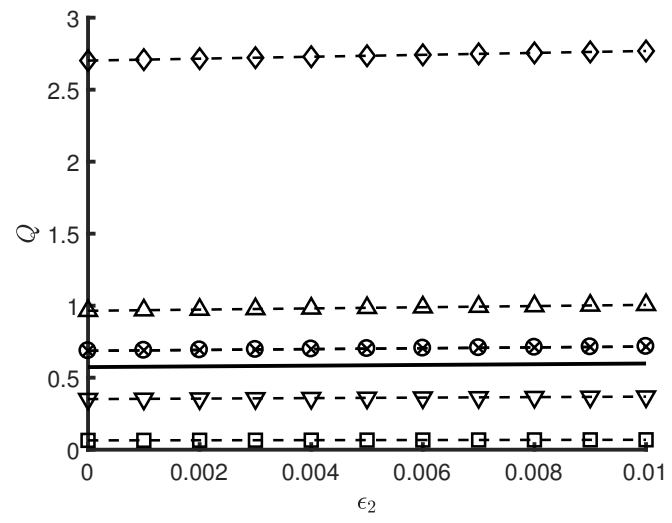

(a) Total flux

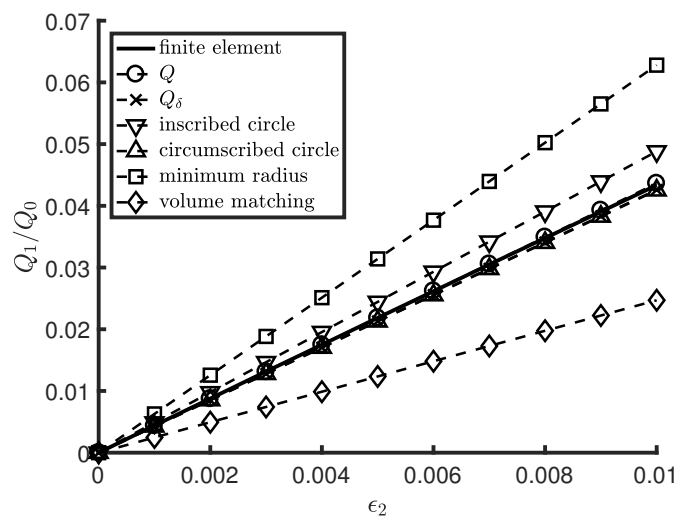

(b) Relative flux increase due to slip

FIG. 7: Comparison between the different flux estimation methods for channel 3, showing a) the total flux through channel 3 , and b) the relative flux increase in the presence of partial slip along the surface of the channel. 
accurately determine the conductivities of the pore throats within the sample as a function of the slip along the surface.

Traditionally, network modelling of a rock sample is performed by assuming that each throat can be represented by a cylindrical tube, with an effective radius determined by the complex geometry of the throat. Various methods are used to estimate the effective radius of the cylinder. Thovert et al. (1993) and Bentz \& Martys (1994) define the radius to be given by the largest sphere which could pass through the throat, which is equivalent to the minimum radius method described in Section 3.3. Al-Kharusi \& Blunt (2008) define the radius using the volume matching method described in Section 3.3. Sisavath et al. (2001) compare the Aissen approximation and the hydraulic radius approximation methods, which we will discuss further in sections 4.1 and 4.2, respectively.

Koplik et al. (1984) construct a network of uniform elliptical channels, with the area and the ratio of the maximum to minimum span matching that of the pore cross section. One of the benefits of studying slip flow through elliptical channels as opposed to circular channels is the ability to vary the circumference and the area of the channel independently. This is useful when trying to estimate the flux through an irregularly shaped channel, as it is important to be able to determine both the total flux through the channel and the relative increase in flux due to slip. By introducing two degrees of freedom, $r_{a}$ and $r_{b}$, we are guaranteed that there exists an elliptic cross section which agrees with both the leading-order and first-order flux through the irregularly shaped channel.

In this section, we briefly discuss how the results obtained in Section 3 can be used to extend current approximations of flow through irregular channels to include slip and varying cross section. We then compare the different approaches with the numerically computed solution for flow through an irregularly-shaped channel with varying cross section taken from a sample of porous rock.

\subsection{Aissen approximation}

The Aissen approximation (Aissen, 1951) is a method used to estimate the flux through a uniform channel, assuming that there is no slip along the surface. If we take $r_{a}$ and $r_{b}$ to be the radii of the smallest circumscribed circle and largest inscribed circle, respectively, of the channel, and $A$ to be the cross-sectional area, then the Aissen approximation for the flux is

$$
Q_{A i}=\frac{A^{2}}{4 \pi} \frac{r_{a} r_{b}}{r_{a}^{2}+r_{b}^{2}} .
$$

Setting $A=\pi r_{a} r_{b}$, we can rewrite (3.33) as

$$
Q_{A i} \sim Q_{A i}^{(0)}+\varepsilon_{2} Q_{A i}^{(1)} \sim\left\{\int_{0}^{1}\left[\frac{A^{2}}{4 \pi} \frac{r_{a} r_{b}}{\left(r_{a}^{2}+r_{b}^{2}\right)}\left(1+\frac{2 \sqrt{2} \varepsilon_{2} \sqrt{r_{a}^{2}+r_{b}^{2}}}{r_{a} r_{b}}\right)\right]^{-1} \mathrm{~d} z\right\}^{-1}
$$

which is an extension of (4.1) to slip flow through channels with varying cross section.

The Aissen approximation is exact for circular and elliptical channels, and accurate to within $10 \%$ for square, triangular, rectangular, and semi-circular channels (Sisavath et al., 2001). However, a disadvantage of this method is that calculating $r_{a}$ and $r_{b}$ requires some computational effort for irregularly shaped channels.

\subsection{Hydraulic radius approximation}

Another method used to approximate to flow through irregularly shaped channels is the hydraulic radius approximation in which $Q$ is assumed to satisfy $Q \approx \frac{1}{2} A R_{H}^{2}$, where $A$ is the area of the cross section, and $R_{H}$ is the hydraulic radius, defined to be $R_{H}:=A / C$ where $C$ is the circumference of the cross section. We reformulate (3.33) in terms of the area and circumference of the channel cross section, in the limit $\delta \rightarrow 0$, to 


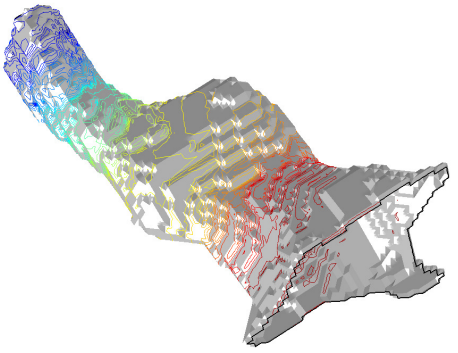

(a) Pore throat extracted from sample

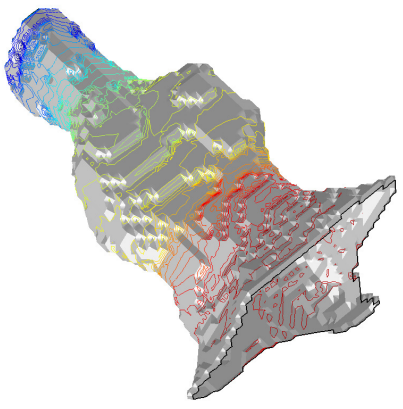

(b) 'Straightened' pore throat

FIG. 8: Data extracted from a Berea sandstone sample using X-ray microtomography, provided by Imperial College London. Figure (a) depicts the pore throat extracted from the sample, and Figure (b) depicts the pore throat with the shape modified such that the central axis is straight.

find that

$$
Q_{H} \sim Q_{H}^{(0)}+\varepsilon_{2} Q_{H}^{(1)} \sim\left\{\int_{0}^{1}\left[\frac{A^{3}}{2 C^{2}}\left(1+2 \varepsilon_{2} \frac{C}{A}\right)\right]^{-1} \mathrm{~d} z\right\}^{-1}=\left\{\int_{0}^{1}\left[\frac{A R_{H}^{2}}{2}\left(1+\frac{2 \varepsilon_{2}}{R_{H}}\right)\right]^{-1} \mathrm{~d} z\right\}^{-1},
$$

where we have used Euler's series expansion for the circumference of an ellipse, $C \sim \pi \sqrt{2\left(r_{a}^{2}+r_{b}^{2}\right)}\left(1+O\left(\delta^{2}\right)\right)$ (Euler, 1774). We observe that (4.3) is the extension of the hydraulic radius approximation to slip flow through a channel of varying cross section. Although it is usually easier to implement, the hydraulic radius approximation is often less accurate than the Aissen approximation (Sisavath et al., 2001).

\subsection{Comparing methods}

To compare the accuracy of the different methods on an irregular channel, we compare the flux estimated by each method with the finite element solution for flow through a pore throat. The example used in this paper is extracted from a Micro CT image of a Berea sandstone provided by Imperial College London. We have smoothed the voxels of the pore throat to reduce the effect of flow trapping in the corners. Figure 8a shows the extracted pore throat after smoothing.

To find the minimum circumscribed circle, maximum inscribed circle, area, and circumference at each cross section along the length of the throat, we suppose that the central axis of the throat lies along the $z$ axis, and we extract the properties from the data in the $(x, y)$ plane at each $z$. This will introduce some error, since the central axis of the throat is curved. To investigate the extent of this error, we also consider a 'straightened' version of the pore throat, depicted in Figure 8b, for which the cross section at each $z$ has been shifted such that the centroid of the cross section lies at $(x, y)=\mathbf{0}$. The central axis of the straightened pore throat is a straight line, with the cross section at each $z$ being identical to the unaltered pore throat. The aspect ratio of the throat is $\varepsilon_{1}=0.36$, the minimum and maximum eccentricity, as measured by the inscribed and circumscribed circle at each point along its length, are $e_{\min }=0.56$ and $e_{\max }=0.93$, respectively, and the minimum and maximum value of $\delta$ along the length of the throat are $\delta_{\min }=0.19$ and $\delta_{\max }=0.76$, respectively.

In Figure 9a we plot the total flux through the throat for the different methods. In terms of the percentage error, the inscribed circle solution is the most accurate, followed by the extended Aissen approximation and extended hydraulic radius approximation. However, the extended Aissen approximation and extended hydraulic radius approximation methods are closest to the straightened pore throat solution. This suggests 


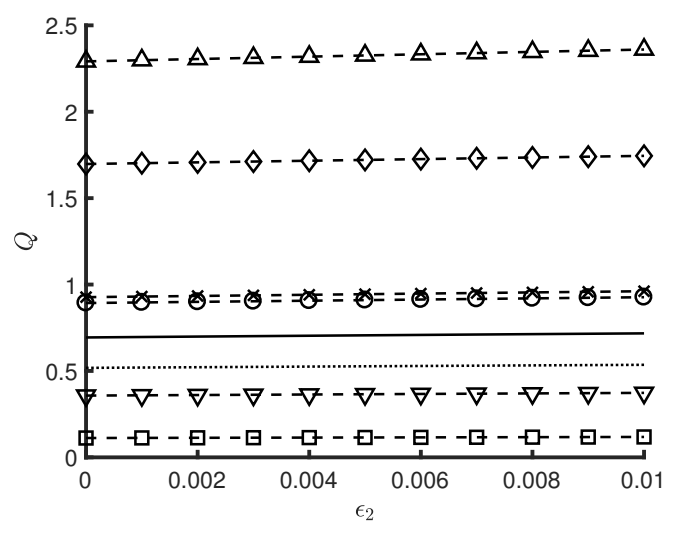

(a) Total flux

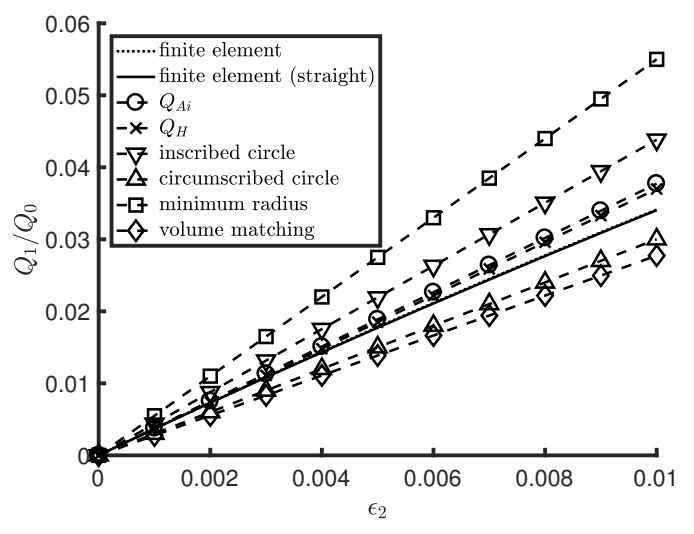

(b) Relative flux increase due to slip

FIG. 9: Comparison between the different flux estimation methods for a) the total flux through the Berea throat, and b) the relative flux increase in the presence of partial slip along the surface of the throat.

that the torsion of the pore throat has a significant affect on the total flux, and introduces noticeable error. In order to correct this issue, a method such as the medial axis method (Lindquist et al., 1996) needs to be applied to find the correct central axis, and the cross section perpendicular to that axis should be considered. Additionally, a mathematical model for the elliptic channel in which the central axis is curved needs to be developed. However, these considerations are beyond the scope of this paper.

We see that the difference between the extended Aissen approximation and extended hydraulic radius approximation is small, and, given the typical error observed in estimating the flux using the different methods, the two approaches can likely be used interchangeably. The approach to use for a particular application will depend on the available data. It is not surprising that the two approaches produce a similar result given that both assume that the channel can be approximated by a varying elliptical cross section. The only difference between the two approaches is that the extended Aissen approximation estimates the semi-major and semi-minor axes using the minimum circumscribed circle and maximum inscribed circle, respectively, and the extended hydraulic radius approximation calculates the axes such that the area and circumference of the ellipse match that of the irregular cross section. The circumscribed circle solution significantly overestimates the total flux. The two methods currently used in practice, minimum radius and volume matching, are the least accurate.

In Figure 9b we plot the relative flux increase due to slip. The extended hydraulic radius method is the most accurate, followed by the extended Aissen approximation. The inscribed radius method and circumscribed radius method overestimate and underestimate, respectively, the relative flux increase. The two methods currently used in practice, minimum radius and volume matching, overestimate and underestimate, respectively, the relative flux increase by the greatest amount. The relative flux increase for the unaltered pore and straightened pore are nearly identical, suggesting that tortuosity primarily affects the leading-order flux. For both the total flux and the relative flux increase, the extended Aissen approximation and extended hydraulic radius methods are significantly more accurate than the methods used currently, the volume matching and minimum radius methods.

\section{Conclusion and discussion}

In this paper, we have presented a model for low-Reynolds number slip flow through a channel of varying elliptic cross section. We solved the system of equations using elliptical coordinates, and we assumed that the aspect ratio of the channel was small. We included slip in the model by supposing that the velocity on the surface of the channel was proportional to the shear stress on the boundary. We assumed that the slip length 
was small in comparison to the width of the channel, so that the problem could be solved as a power series in the dimensionless slip length.

In Section 3.1, we derived an expression for the flux through the channel, (3.29). We found that, since the shape of the cross section varies along its length, the flux is given by the harmonic mean of the equivalent flux through a uniform channel at each cross section. The leading-order (in $\varepsilon_{2}$ ) flux is the harmonic mean of the standard result for quasi-Poiseuille flow through an elliptic channel, and the first-order flux is written in terms of an elliptic integral, which, to solve numerically, needs to be computed at each cross section along the length of the channel.

In Section 3.2, we expanded the elliptic integral in terms of $\delta=\left(r_{a}^{2}-r_{b}^{2}\right) /\left(r_{a}^{2}+r_{b}^{2}\right) \ll 1$, to find a simplified expression for the flux, (3.33). We compared the flux increase due to slip given by the simplified expression for the flux, $Q_{\delta}^{(1)} / Q_{\delta}^{(0)}$, with the full expression, $Q^{(1)} / Q^{(0)}$, and found that there is agreement to within $16.8 \%$, even for very eccentric ellipses. The difference is within $5 \%$ for $\delta<0.55$, which corresponds to $r_{b}<r_{a}<1.8 r_{b}$, and within $1 \%$ for $\delta<0.24$, which corresponds to $r_{b}<r_{a}<1.3 r_{b}$.

In section 3.3, we computed the flux through three elliptic channels. We compared the flux calculated by two existing methods, the 'volume matching' and 'minimum radius' methods, with $Q, Q_{\delta}$, the 'inscribed circle' and 'circumscribed circle' methods, and the numerical finite element solution. We found that $Q$ and $Q_{\delta}$ are in excellent agreement with the finite element solution, the volume matching method consistently overestimates the total flux, and the minimum radius method consistently underestimates the total flux. Furthermore, the volume matching method consistently underestimates the relative flux increase due to slip, and the minimum radius method consistently overestimates the relative flux increase.

In section 4.3, we computed the flux through a pore throat extracted from a Micro-CT image of a Berea sandstone, as calculated by the different methods. We conclude that the methods which approximate the pore throat using a channel with varying elliptic cross section, which can be formulated as extensions to the Aissen approximation and hydraulic radius approximation (equations (4.2) and (4.3), respectively), are typically the most accurate methods. More importantly, the elliptic approaches are less susceptible to the systematic errors observed when applying the other methods. In particular, the methods currently used in practice, the minimum radius method and volume matching method, consistently underestimate and overestimate, respectively, the total flux. Additionally, the minimum radius method consistently overestimates the relative flux increase due to slip, and the volume matching method consistently underestimates the flux increase due to slip. By applying either of these methods to an entire network of pore throats, the network model is likely to inaccurately estimate the permeability of the porous structure due to the cumulative effect of consistently overestimating or underestimating the flux. If a network constructed in this way is used to study applications that depend on the slip on the solid surface, such as low salinity waterflooding (Wilmott et al., 2018), the model is likely to miscalculate the effect of the slip length on flow through the structure.

The main limitation of the model presented in this paper is the assumption that the central axis is straight. While this will give a reasonable estimate for the flux through a slightly curved channel, there are many applications where this is not the case. In particular, we observe that the elliptic approximations overestimate the flux through the pore throat extracted from the Berea sandstone. This is partly due to the fact the flux through the throat is reduced as a result of the central axis being curved, since the error is reduced when comparing the elliptic approximations with the straightened pore. Further work needs to be done to generalise the model for curved channels. However, the model presented in this paper improves on current methods used to estimate the flux through an irregularly shaped channel, and it is the aim of future work to apply the results to a network of pore throats in order to accurately and efficiently estimate the flow through a porous structure.

\section{Funding}

This work was supported by Engineering and Physical Sciences Research Council and BP in the form of a CASE conversion, grant number EP/M50659X/1. 


\section{Acknowledgements}

We would like to thank Tim Totton and Bilal Rashid for their continued support throughout the project, in particular for their advise regarding industrial methods and working with rock sample data.

\section{REFERENCES}

Aissen, M. I. (1951) On the estimation and computation of torsional rigidity. Dept. of Mathematics, Stanford University.

Al-Kharusi, A. S. \& Blunt, M. J. (2008) Multiphase flow predictions from carbonate pore space images using extracted network models. Water Resources Research, 44(6).

Al-Raoush, R. I. \& Willson, C. S. (2005) Extraction of physically realistic pore network properties from three-dimensional synchrotron X-ray microtomography images of unconsolidated porous media systems. Journal of Hydrology, 300(1), 44-64.

Bahrami, M., Tamayol, A. \& Taheri, P. (2009) Slip-flow pressure drop in microchannels of general cross section. Journal of Fluids Engineering, 131(3), 031201.

Bentz, D. P. \& Martys, N. S. (1994) Hydraulic radius and transport in reconstructed model three-dimensional porous media. Transport in Porous Media, 17(3), 221-238.

Duan, Z. \& Muzychka, Y. S. (2007) Slip flow in elliptic microchannels. International Journal of Thermal Sciences, 46(11), 1104-1111.

Euler (1774) Nova series infinita maxime convergens perimetrum ellipsis exprimens. Novi Commentarii Academiae Scientiarum Petropolitanae, 18, 71-84.

Ghosal, S. (2002) Lubrication theory for electro-osmotic flow in a microfluidic channel of slowly varying cross-section and wall charge. Journal of Fluid Mechanics, 459, 103-128.

Koplik, J., Lin, C. \& Vermette, M. (1984) Conductivity and permeability from microgeometry. Journal of Applied Physics, 56(11), 3127-3131.

Lager, A., Webb, K., Black, C., Singleton, M. \& Sorbie, K. (2008) Low salinity oil recovery-An experimental investigation. Petrophysics, 49(1), 28-35.

Lee, S., Webb, K., Collins, I., Lager, A., Clarke, S., O’Sullivan, M., Routh, A. \& Wang, X. (2010) Low salinity oil recovery: Increasing understanding of the underlying mechanisms. In SPE improved oil recovery symposium. Society of Petroleum Engineers.

Lindquist, W. B., Lee, S. M., Coker, D. A., Jones, K. W. \& Spanne, P. (1996) Medial axis analysis of void structure in three-dimensional tomographic images of porous media. Journal of Geophysical Research: Solid Earth, 101(B4), 8297-8310.

Myint, P. C. \& Firoozabadi, A. (2015) Thin liquid films in improved oil recovery from low-salinity brine. Current Opinion in Colloid \& Interface Science, 20(2), 105-114.

Sheng, Q. \& Thompson, K. (2016) A unified pore-network algorithm for dynamic two-phase flow. Advances in Water Resources, 95, 92-108.

Sisavath, S., Jing, X. \& Zimmerman, R. W. (2001) Laminar flow through irregularly-shaped pores in sedimentary rocks. Transport in Porous Media, 45(1), 41-62.

Thovert, J. F., Salles, J. \& Adler, P. M. (1993) Computerized characterization of the geometry of real porous media: their discretization, analysis and interpretation. Journal of Microscopy, 170(1), 65-79.

Wilmott, Z. M., Breward, C. J. W. \& Chapman, S. J. (2018) The effect of ions on the motion of an oil slug through a charged capillary. Journal of Fluid Mechanics, 841, 310-350. 